\title{
Assessment of intra-tumoral karyotypic heterogeneity by interphase cytogenetics in paraffin wax sections
}

\author{
S A Southern, C S Herrington
}

\begin{abstract}
Aim-To analyse the effect of sectioning on the assessment of karyotypic heterogeneity by interphase cytogenetics in paraffin wax embedded normal squamous epithelium and to apply the principles derived to invasive cervical carcinoma. Methods-Normal male $(n=5)$ and female $(n=5)$ squamous epithelia were hybridised with peri-centromeric repeat probes specific for chromosomes $X$ (DXZ1) and 17 (D17Z1) individually and in combination to assess the effect of sectioning on mono-, di-, tri-, and tetrasomic populations. Section thickness, interobserver variation and variation between different areas of the epithelium were evaluated. Invasive squamous carcinomas of the cervix $(n=5)$ were then hybridised with the DXZ1 probe and intratumoral heterogeneity was assessed by comparison of signal distributions obtained from different areas.
\end{abstract}

Results-The optimum section thickness for the assessment of normal epithelium was $6 \mu \mathrm{m}$. Variation in the expected signal number in the range $1-4$ did not introduce artefactual heterogeneity at this section thickness. The sensitivity of this approach for the detection of minor subpopulations was calculated to be $13-16 \%, 17-18 \%$ and $10-11 \%$ for mono-, tri- and tetrasomic populations, respectively. Karyotypic heterogeneity was detected in two of the five tumours and, in one case where the populations where clustered morphologically, a minor population representing $18 \%$ was identified.

Conclusions-Interphase cytogenetic analysis of sections from paraffin wax embedded material can be used for the detection of minor subpopulations in tumours. This approach will be of particular value in the assessment of the relation between human papillomavirus infection and tumour karyotype and in the analysis of intraepithelial neoplasia.

University of Liverpool, Department of Pathology, Duncan Building, Royal Liverpool University Hospital,

Liverpool L69 3BX

Correspondence to: Dr C S Herrington.

Accepted for publication 9 July 1996

$$
\begin{aligned}
& \text { Keywords: interphase } \\
& \text { some, heterogeneity. }
\end{aligned}
$$

Interphase cytogenetics involves karyotypic analysis by the demonstration of specific DNA sequences in interphase nuclei. ${ }^{2}$ This has several advantages over the conventional approach, which is based on the analysis of meta- phase chromosomes. Cell culture is not required and hence cytological and histological material can be analysed directly. The technique is quicker and does not require cytogenetic expertise for the analysis of results as, given appropriate specificity of controls and probe characterisation, the presence of signal can be used to indicate the appropriate target sequence. This approach also has the potential for detection of subpopulations within karyotypically complex tissues and thus can be applied to the investigation of intra-tumoral heterogeneity and of mosaicism.

Numerical chromosome abnormalities can be demonstrated by in situ hybridisation with peri-centromeric repeat probes. ${ }^{3}$ This approach can be used to analyse individual chromosome aneusomy in tumour derived material, such as cell lines ${ }^{4}$ and fine needle aspirates. ${ }^{5}$ The application of this approach to paraffin wax embedded material is more difficult as, if whole nuclei are extracted from thick sections, ${ }^{6}$ morphological correlation is not possible but, if thin sections are used, morphological assessment is achieved at the expense of nuclear truncation as a result of sectioning. ${ }^{7-13}$ However, morphological correlation is a prerequisite for the analysis of intratumoral heterogeneity by this technique and therefore it is important to assess the information which can reliably be gained from such an approach. Although there are studies in which karyotypic heterogeneity within tumours has been demonstrated, there has been no systematic approach to the assessment of the reliability of the data produced, of the effect of sectioning in archival material or of the sensitivity of this method for the detection of minor subpopulations.

The aim of the present study was therefore to analyse the effect of sectioning on the assessment of karyotypic heterogeneity in paraffin wax embedded material. In order that data derived from the analysis of invasive tumours, which are likely to have a variably complex karyotype, could be interpreted, the effect of nuclear sectioning was modelled using normal squamous epithelium which has a stable, normal karyotype. The principles derived from this analysis were then applied to invasive cervical carcinomas.

\section{Methods}

CHOICE OF CASES

The following three groups of cases were selected from the diagnostic files of the Royal Liverpool University Hospital: (1) five normal 
cervices from hysterectomy specimens from patients with no known history of cervical neoplasia; (2) five biopsy specimens of normal non-keratinising oral squamous epithelium from male patients with no history of malignant disease; and (3) five invasive cervical squamous carcinomas.

PROBES

The chromosome specific probes used were biotinylated D17Z1 and DXZ1 (Oncor, Gaithersburg, Maryland, USA). These probes label peri-centromeric repetitive sequences and have been localised previously to the appropriate chromosome. ${ }^{4}$ The DYZ3 probe (Oncor, USA) was used to confirm the presence of a Y chromosome in biopsy specimens from male patients.

\section{INTERPHASE CYTOGENETICS}

The method used was based on that of Hopman et al. ${ }^{3}$ Briefly, paraffin wax sections were dewaxed and pretreated for 10 minutes in $1 \mathrm{M} \mathrm{NaSCN}$ at $80^{\circ} \mathrm{C}$. Sections were washed in water and then digested with $0.4 \%(\mathrm{w} / \mathrm{v})$ pepsin (Sigma, Poole, Dorset, UK; P7012) in $0.2 \mathrm{M} \mathrm{HCl}$ for 20 minutes at $37^{\circ} \mathrm{C}$. Following washes in water, sections were air dried and then the probe was applied at a concentration of $1 \mathrm{ng} / \mu \mathrm{l}$ in $2 \times \mathrm{SSC}, 10 \%$ dextran sulphate, $60 \%$ formamide, and $1 \mu \mathrm{g} / \mathrm{ml}$ sheared salmon sperm DNA. Denaturation was carried out at $80^{\circ} \mathrm{C}$ for eight minutes and hybridisation at $37^{\circ} \mathrm{C}$ overnight. After hybridisation, sections were washed with $60 \%$ formamide, $2 \times$ SSC, pre-equilibrated to $\mathrm{pH} 7.0$ at $42^{\circ} \mathrm{C}$ for $20 \mathrm{~min}$ utes and then phosphate buffered saline (PBS) containing $3 \%(\mathrm{w} / \mathrm{v})$ bovine serum albumin (BSA) and $0.05 \%$ Tween 20 (buffer A) for 20 minutes at room temperature. Detection was performed by sequential incubation at $37^{\circ} \mathrm{C}$ in monoclonal anti-biotin (diluted 1 in 100), peroxidase conjugated rabbit anti-mouse (diluted 1 in 80) and peroxidase conjugated swine antirabbit (diluted 1 in 100) for 30 minutes each. Washes were carried out in buffer A. Signal was developed using 3,3'-diaminobenzidine (DAB)/ $\mathrm{H}_{2} \mathrm{O}_{2}$. Sections were counterstained with haematoxylin and mounted in DPX.

\section{INTERPRETATION}

The number of signals per nucleus was counted at a total magnification of $\times 630$ and the following rules observed: (1) all signals were counted; (2) overlapping nuclei were not counted; and (3) split signals, as defined previously, ${ }^{4}$ were counted as single signals. Signal number was recorded for each nucleus individually in the order of counting. Where appropriate, slides were assessed independently by two observers.

STATISTICAL ANALYSIS

Distributions were assessed using the MannWhitney $U$ test, corrected for tied values, and the $\chi^{2}$ test for trend. ${ }^{14}$ Heterogeneity was assessed by plotting dot number per nucleus against the order in which the nuclei were counted in order to retain morphological information.

\section{Results}

NORMAL SQUAMOUS EPITHELIUM

Section thickness

The effect of section thickness was assessed by hybridising the DXZ1 probe to 4,6 and $8 \mu \mathrm{m}$ sections from each of the five normal cervices. Optimum signal distributions were obtained in all cases with $6 \mu \mathrm{m}$ and $8 \mu \mathrm{m}$ sections (fig 1), with $58-68 \%$ of nuclei containing two signals. No difference was observed between distributions obtained by analysis of 300 nuclei from 6 $\mu \mathrm{m}$ and $8 \mu \mathrm{m}$ sections, but $8 \mu \mathrm{m}$ sections were less easily interpreted because of greater nuclear superimposition, particularly in the basal and para-basal layers. Therefore, $6 \mu \mathrm{m}$ thick sections were used in the remainder of the study.

\section{Interobserver variation}

Two hundred nuclei from each of three separate areas from each of the five normal cervices were counted independently by two observers. No significant difference was found between the distributions derived in each case (fig 2).

\section{Effect of expected signal number}

A single signal was obtained in the majority of cells with the DYZ1 probe in male but not in female epithelia. In order to model the effect of sectioning on cell populations with different karyotypes, normal male and female squamous epithelia were hybridised with DXZ1 and $\mathrm{D} 17 \mathrm{Z1}$, both individually and in combination. This approach produced populations with expected dot numbers of one (male epithelium, DXZ1 alone), two (female epithelium, DXZ1 alone), three (male epithelium, DXZ1 and $\mathrm{D} 17 \mathrm{Z1}$ ), and four (female epithelium, DXZ1 and D17Z1) (fig 3). Two hundred nuclei were counted from each of three separate areas from each of the five cases and frequency distributions were derived using the first 100, 150 and all 200 nuclei. Hybridisation with $\mathrm{DXZ1}$ alone produced a single signal in $74-81 \%$ of nuclei in male epithelium, with two signals being identified in $0-4 \% ; 58-69 \%$ of

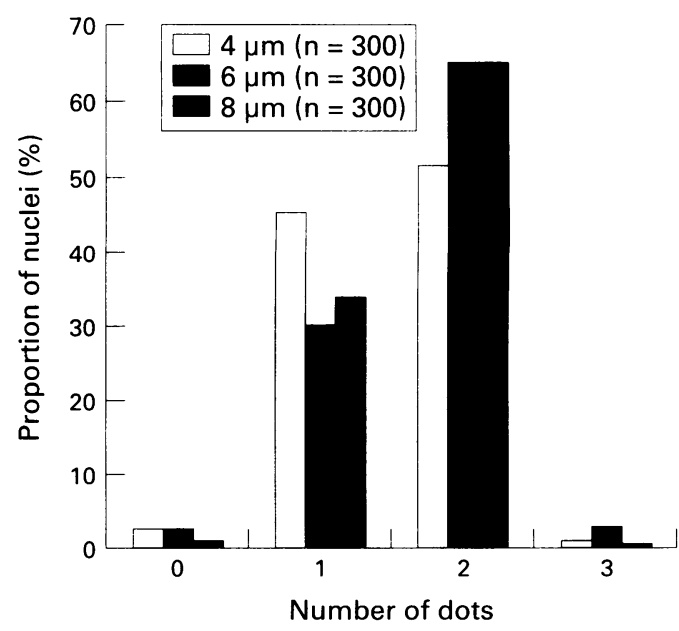

Figure 1 Relation between section thickness and signal distribution in normal epithelium. Although optimum results were obtained with both $6 \mu \mathrm{m}$ and $8 \mu \mathrm{m}$ sections in each case, $6 \mu \mathrm{m}$ sections were interpreted more easily. 

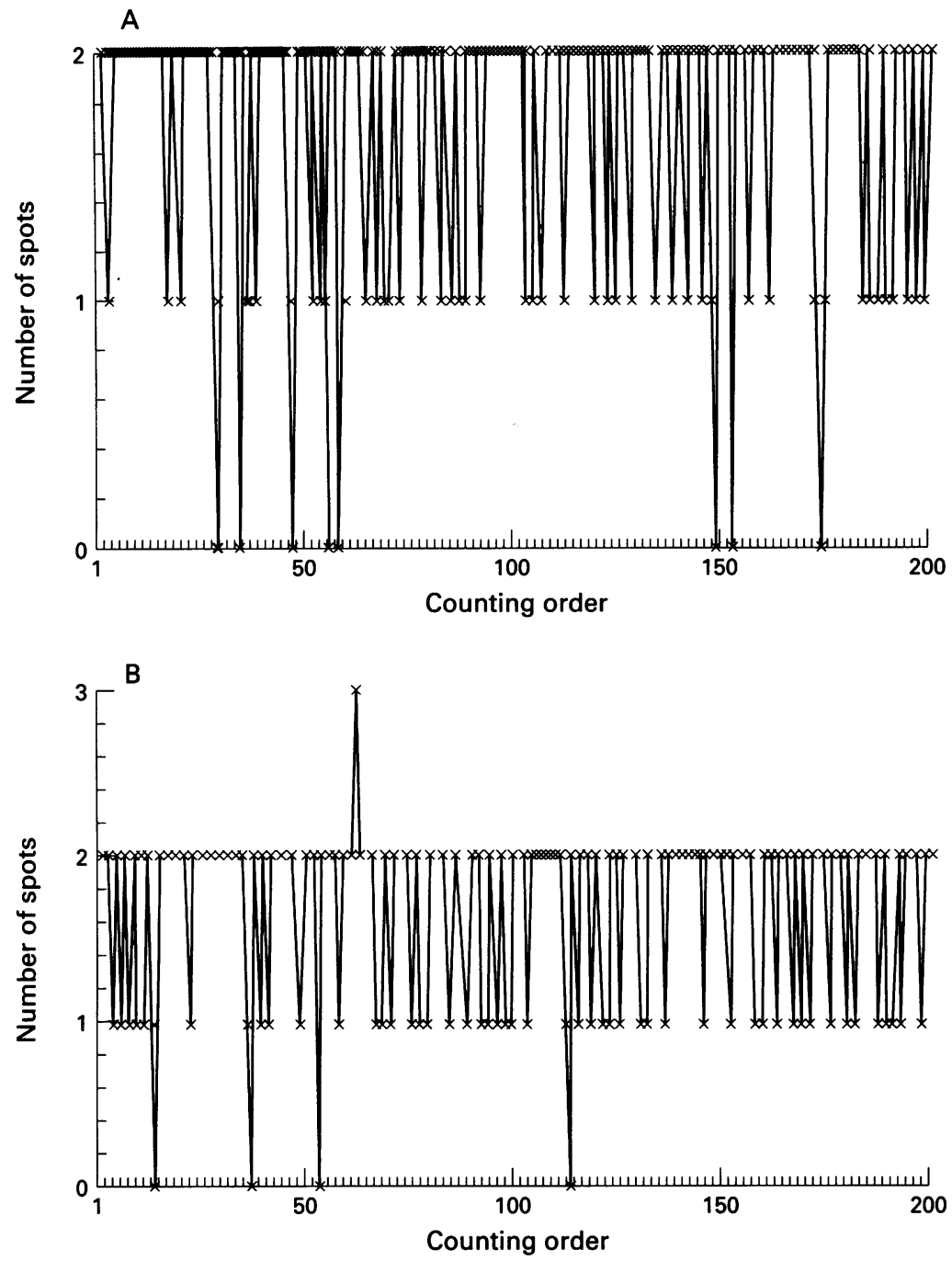

Figure 2 Plotting nuclear signal number against the order in which the nuclei were counted in normal epithelium leads to some clustering of nuclei containing the same signal number, but there is no significant difference (Mann-Whitney $U$ test) between the plots obtained by two independent observers. $A$, observer $1 ; B$, observer 2 .

nuclei contained two signals and $0-2 \%$ three signals in female epithelium. Variation between cases after cohybridisation (fig 4) was related to hybridisation efficiency as there was a direct relation between the distributions obtained with the individual probes and that with the combined probe. For example, in case 1 (shown in fig 4B) $65 \%$ of nuclei contained two signals with the individual probes compared with only $58 \%$ with each probe in case 2 . However, distributions derived from the three separate areas counted did not differ significantly in any case when hybridised with one or both probes. This indicates that sectioning does not introduce apparent heterogeneity artefactually in cell populations with up to four expected signals. The number of nuclei counted had no effect over the range 100-200, indicating that deviation, even in relatively small groups of nuclei, is not introduced by sectioning.

This set of experiments also permits the assessment of the sensitivity of this approach for the detection of minor subpopulations. The presence of such populations within otherwise normal epithelium was modelled by a weighted combination of the appropriate distributions from each case, followed by comparison with the disomic distribution obtained with D17Z1 .
The male epithelia were used to derive sensitivities for the detection of monosomy and trisomy, and the female epithelia for tetrasomy. For example, for the evaluation of trisomy, the distribution obtained with both DXZ1 and D17Z1 in male epithelium was combined with that obtained with $\mathrm{D} 17 \mathrm{Z1}$ alone and the resultant distribution compared using the $\chi^{2}$ test for trend with the D17Z1 distribution. This shows that a monosomic population can be detected if it represents $13-16 \%$ of the total, a trisomic population if it represents $17-18 \%$ and a tetrasomic population if it represents $10-11 \%$.

\section{CERVICAL CARCINOMAS}

Each of five cervical carcinomas was hybridised with the $\mathrm{DXZ1}$ probe alone (fig 5). In two cases normal epithelium was present in the sections and gave distributions with $60-65 \%$ of nuclei containing two signals; in three cases lymphocytes within the section gave a signal distribution with $65-70 \%$ of nuclei containing two signals, indicating technical adequacy. Signals were counted from 200 nuclei in each of three previously marked areas of all five tumours by two observers. In all cases fewer than $5 \%$ of nuclei contained no signal.

\section{Interobserver variation}

No significant interobserver variation was identified in four of the cases (fig 6), but in one case there was apparent heterogeneity in one area. By plotting the signal number per nucleus as a function of the order in which the nuclei were counted, it was shown that those cells responsible for the discrepancy were clustered morphologically. Review of the slide revealed that observer 1 had counted a tumour focus that observer 2 had not, indicating that cell selection and not differences in signal interpretation was responsible for the variation: this case showed significant intra-tumoral heterogeneity (fig 7A).

\section{Intra-tumoral heterogeneity}

Significant heterogeneity between the three areas counted was statistically demonstrable in two cases and not in three (Mann-Whitney U test; fig 7). Plotting signal number as a function of counting order demonstrated the presence of discrete populations of tumour cells in one case (figs $7 \mathrm{~A}$ and 8 ). The distribution obtained from area 1 is significantly different from that obtained from area $2(p=0.01)$. It can be calculated that the minor population in this case in area 1 represented approximately $18 \%$ of the nuclei counted.

\section{Discussion}

Interphase cytogenetic analysis of paraffin wax embedded material can be carried out in two ways: by extraction of whole nuclei from thick paraffin wax sections; and by using thin paraffin wax sections. The former approach allows archival material to be used but does not permit morphological correlation as tissue architecture is destroyed. Therefore, where such correlation is important, the use of thin sections is mandatory. This approach, how- 

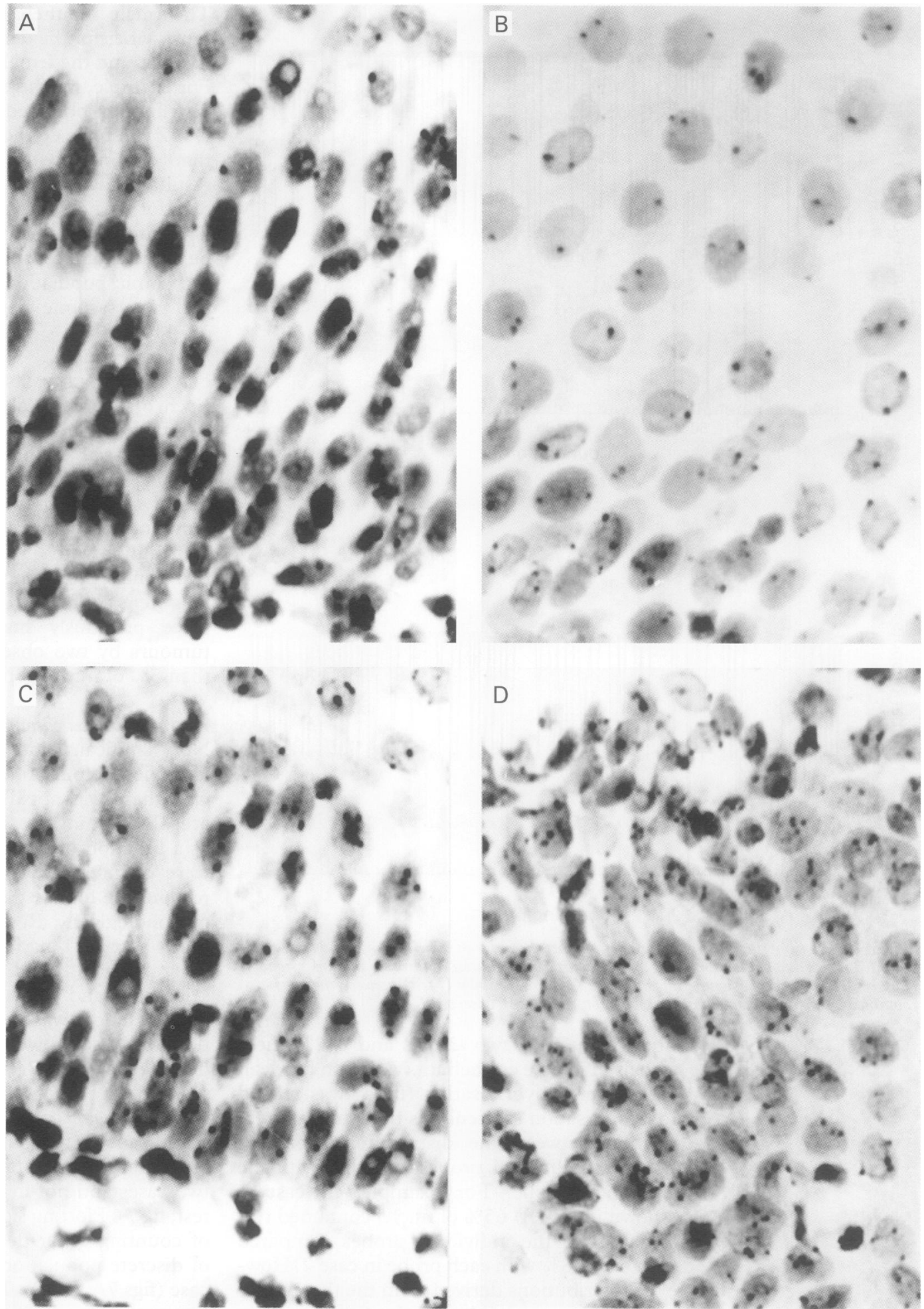

Figure 3 Interphase cytogenetics of normal squamous epithelium. Hybridisation with DXZ1 alone in $(A)$ male and $(B)$ female epithelia gives modal signal numbers of 1 and 2, respectively. Hybridisation with both DXZ1 and D17Z1 gives variable signal numbers but nuclei containing three signals can be identified in male epithelium (C) and four signals in female epithelium (D). Note that care must be taken not to count overlapping nuclei, as shown particularly in (D).

ever, leads to the production of partial nuclear profiles with consequent loss of nuclear material and hence artefactual reduction in genetic content. For interpretation of such preparations, this artefactual effect must be separated from several other variables, including variation in karyotype and in nuclear size.

In this study, the effect of sectioning was explored in two separate ways. In the first approach, section thickness was varied whilst keeping the expected signal number constant in normal squamous epithelium. The choice of section thickness is determined by the conflicting effects of nuclear sectioning and nuclear superimposition. From the data presented here, the optimum thickness in our hands is 6 $\mu \mathrm{m}$ and the proportion of nuclei containing two signals is in the range $58-69 \%$ : this is consistent with data reported by others. ${ }^{7}$ The use of $4 \mu \mathrm{m}$ sections led to a significant reduction in the proportion of normal epithelial cell nuclei containing two signals; $8 \mu \mathrm{m}$ thick sections, although giving an acceptable signal distribution, were more difficult to interpret in 

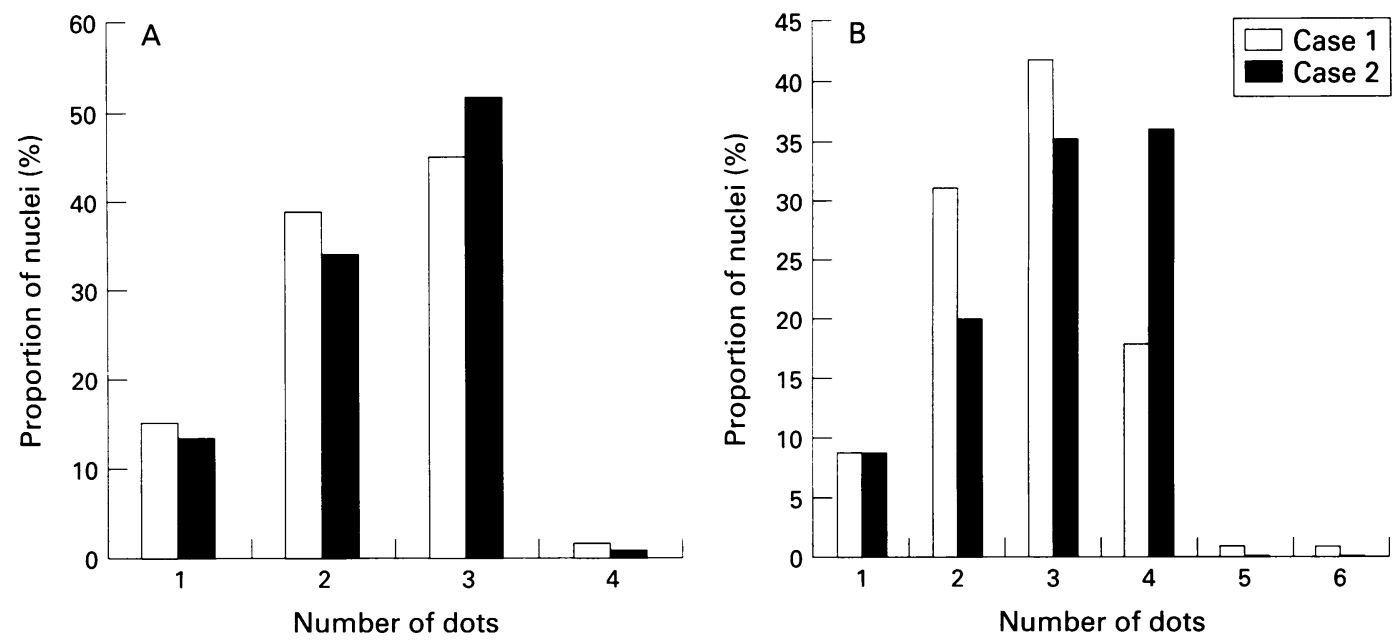

Figure 4 Frequency histograms illustrating the spectrum obtained from normal squamous epithelia after co-hybridisation with DXZ1 and D17Z1 in (A) male and (B) female epithelia. The two cases illustrated in each panel represent the best and worst result obtained. The distributions obtained with the combined probe reflect the hybridisation efficiency obtained using the probes individually (see Results) and demonstrate that the modal peak does not necessarily equal the expected signal number.

view of nuclear overlapping, particularly in the basal layers of the epithelium.

The second approach involved keeping the section thickness constant at $6 \mu \mathrm{m}$ and varying the expected signal number in normal epithelium by hybridising male and female epithelia with alphoid probes specific for chromosomes $\mathrm{X}$ and 17 , both individually and in combination. This demonstrated that there was varia-

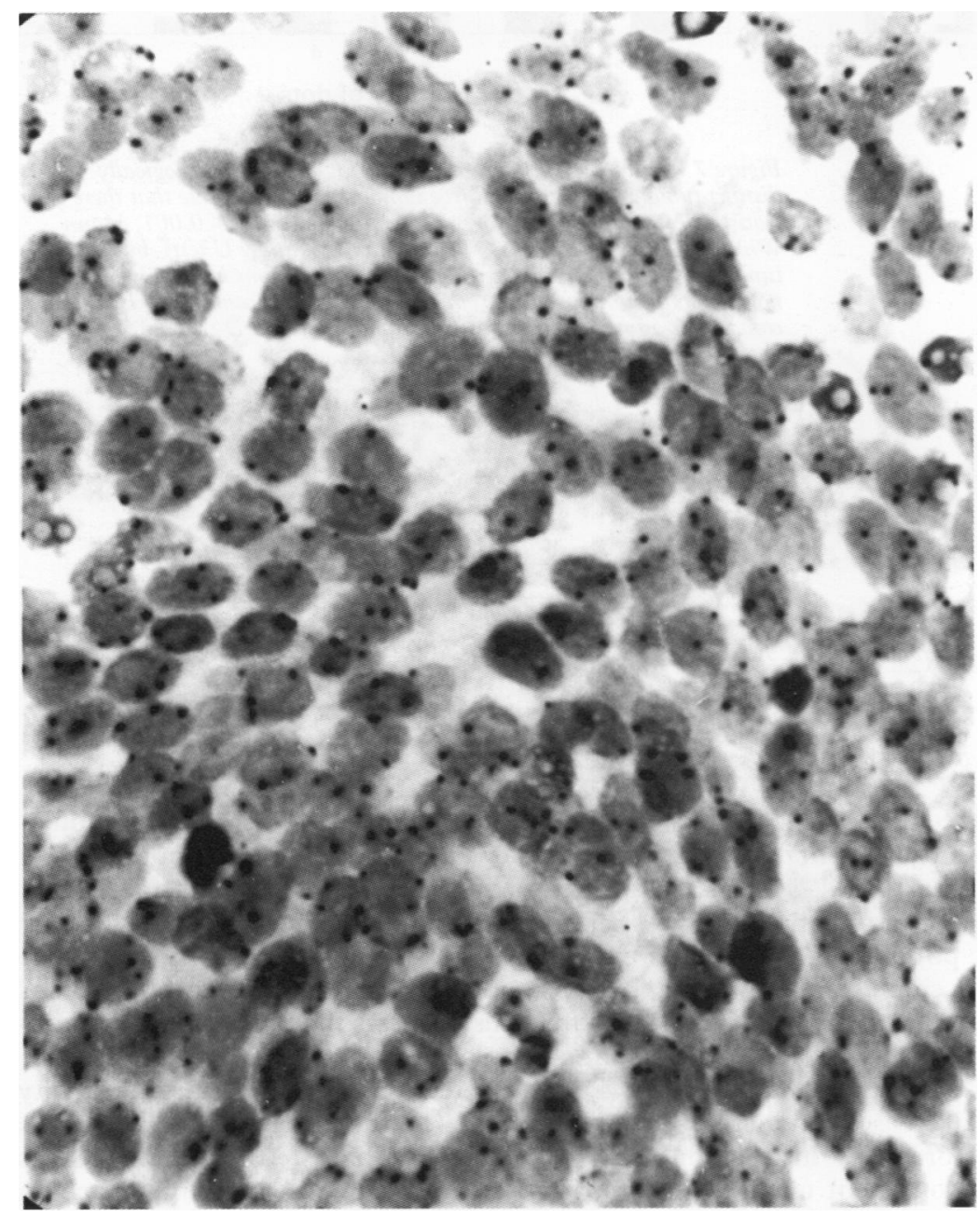

Figure 5 Interphase cytogenetics of an invasive squamous cell carcinoma (fig $7 A$ ) showing that the majority of tumour nuclei contain two or three signals. tion between cases, including normal epithelium, but that, when different areas of the same case were analysed, there was no difference in signal distribution. These results indicate that sectioning may lead to variation in distributions between cases even when the expected signal number is the same. This is most likely the result of a combination of factors, including variation in hybridisation efficiency, differences in fixation and small variations in section thickness. In general, it was found that repeating the hybridisation gave the same distribution, indicating that variation between cases was probably because of fixation differences. This observation indicates that a particular frequency distribution cannot be regarded as indicative of the chromosome number in the intact tissue and is of importance when analysing more complex karyotypes, particularly when there is also variation in nuclear size. However, the observation that there is no variation between areas of the same case indicates that statistically significant heterogeneity is not introduced by sectioning when 100-200 nuclei are assessed from each area.

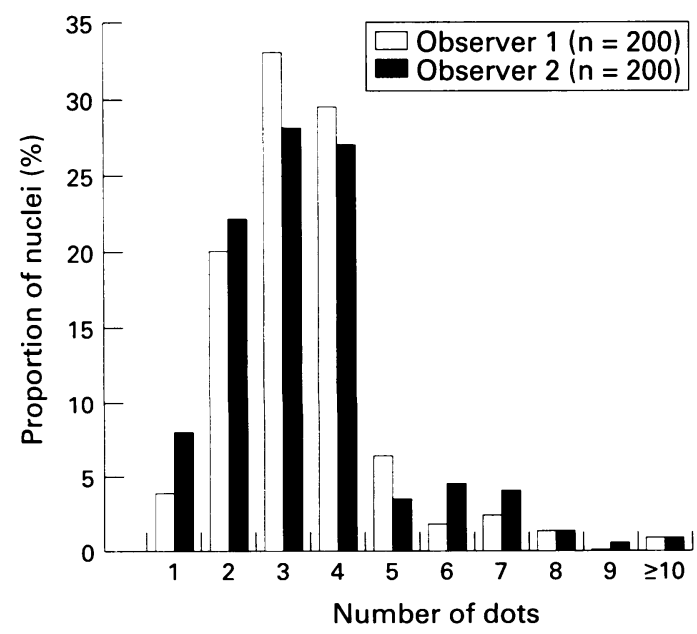

Figure 6 Frequency histogram illustrating that no significant interobserver variation (Mann-Whitney $U$ test) was obtained in a case with a wide range of signal numbers. This is representative of the findings in all cases analysed. 

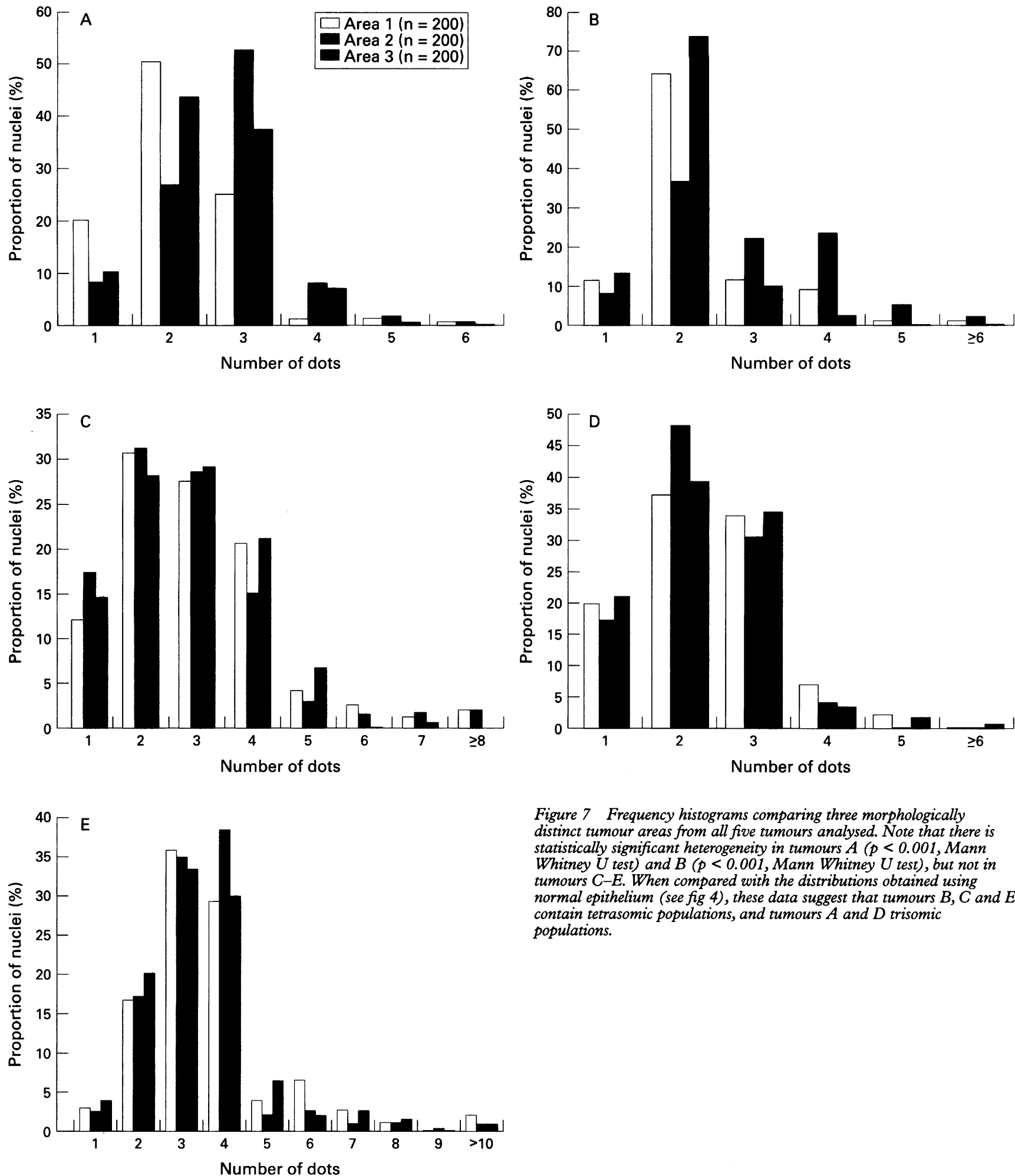

Figure 7 Frequency histograms comparing three morphologically distinct tumour areas from all five tumours analysed. Note that there is statistically significant heterogeneity in tumours $A(p<0.001$, Mann Whitney $U$ test $)$ and $B(p<0.001$, Mann Whitney $U$ test $)$, but not in tumours $C-E$. When compared with the distributions obtained using normal epithelium (see fig 4), these data suggest that tumours $B, C$ and $E$ contain tetrasomic populations, and tumours $A$ and $D$ trisomic populations.

The sensitivity for detection of a trisomic population in a background of normal cells is approximately $17-18 \%$ (that is, $34-36$ cells), $13-16 \%$ for a monosomic population, and $10-11 \%$ for a tetrasomic population. The sensitivities are not affected significantly by the efficiency of hybridisation, as shown by the narrow ranges. This is most likely because variation in efficiency affects both probes equally. However, sensitivity will decrease with increasing nuclear size. Therefore, the distinction between a tumour with consistent aneusomy and an aneusomic subpopulation cannot necessarily be made-for example, a tumour con- sisting of small cells with a trisomic subpopulation could give the same distribution as a purely trisomic tumour with large nuclei. It is therefore more appropriate simply to state that there is a statistically significant difference between normal and tumour cell populations and that this suggests the presence of an aneusomic population.

For the detection of trisomy, the sensitivity derived equates to the presence of three signals in $9-10 \%$ of cells (that is, $18-20$ nuclei). This provides some objective basis for the assertion in some studies that $10-11 \%$ can be used as a cut-off point for the detection of trisomy, ${ }^{13} 1516$ 

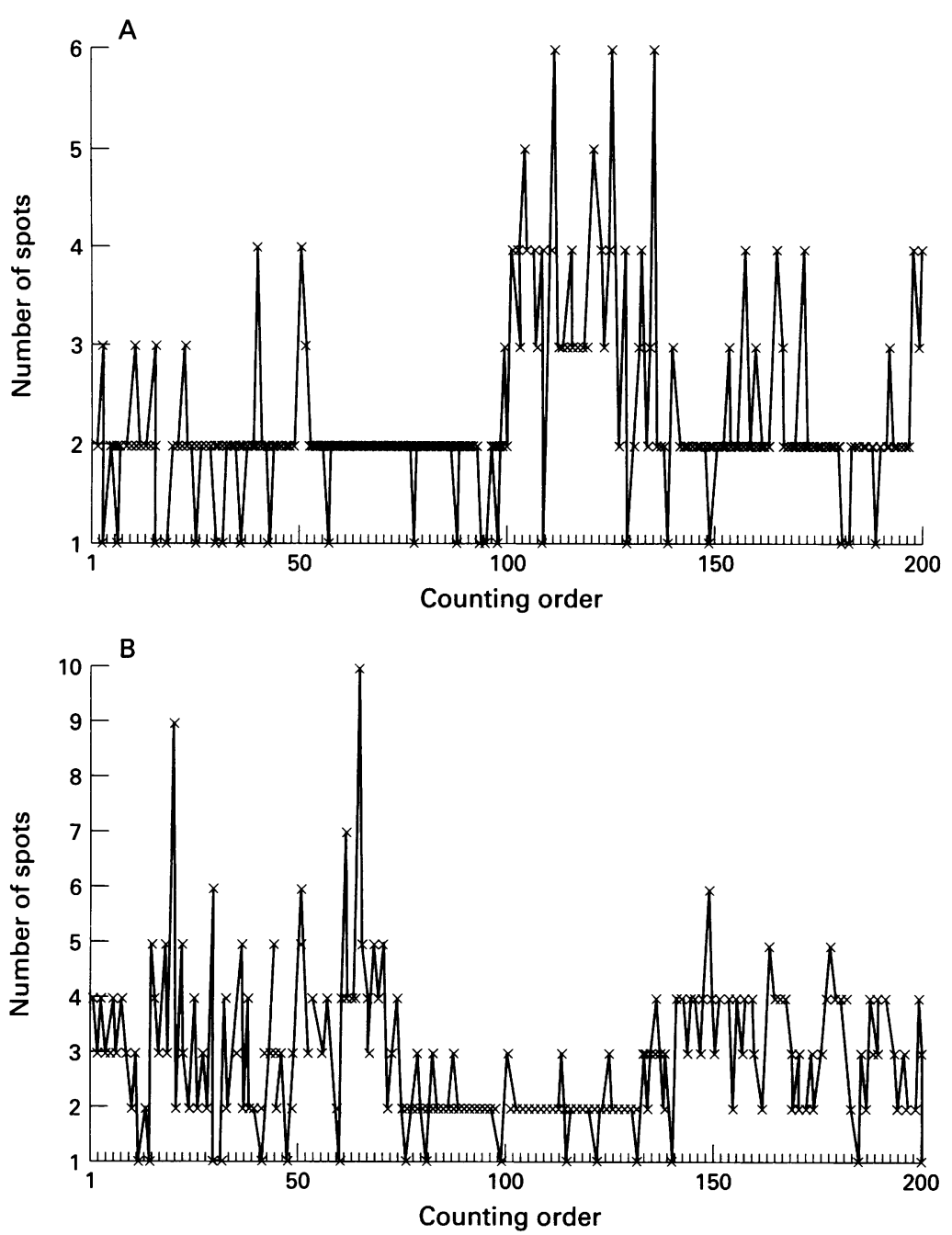

Figure 8 The relation between signal number and counting order in areas $1(A)$ and 2 (B) from the case illustrated in fig $7 B$. Note that separate populations are clearly demonstrated by this approach. obtained from a tumour by comparison with normal epithelium cannot be reliably attributed to monosomy of a proportion of the tumour cells. However, variation within tumours is less affected by this as larger tumour cells tend to contain greater numbers of signals and therefore can be interpreted reliably as heterogeneous. Probes for more than one chromosome can be used to control for variation in nuclear size as such variation affects all probes equally: any differences must therefore result from karyotypic variation and relative chromosome loss can be detected reliably.

In conclusion, therefore, paraffin wax sections can be used to demonstrate intra-tumoral heterogeneity of chromosome number. Increase in expected signal number does not alone introduce artefactual apparent heterogeneity and differences between areas of the same tumour can be interpreted as indicating heterogeneity if variation in nuclear size is taken into account. The approach described here will be particularly useful in the analysis of intraepithelial neoplasia, where morphological correlation is important.

We thank the University of Liverpool for financial support.

1 Herrington CS. Interphase cytogenetics. $\mathcal{f}$ Histotechnol 1994;17:219-34.

2 Poddighe PJ, Ramaekers FC, Hopman AH. Interphase cytogenetics of tumours. $\mathcal{F}$ Pathol 1992;166:215-24

3 Hopman AHN, Poddighe P, Moesker O, Ramaekers FCS Interphase cytogenetics: an approach to the detection of genetic aberrations in tumours. In: Diagnostic molecular pathology: a practical approach. Vol 1. Oxford: Oxford University Press, 1992:141-67.

4 Herrington CS, Cooper K, McGee JO'D. Interphase cytogenetics: analysis of numerical chromosome aberra cytogenetics: analysis of numerical chromosom

5 Herrington CS, Leek RD, McGee JO'D. Correlation of numerical abnormalities of chromosomes 11 and 17 with metastasis of primary breast cancer to lymph nodes. $\mathcal{F}$ Pathol. 1995;176:353-9.

6 Arnoldus EP, Peters AC, Bots GT, Raap AK, van der Ploeg $M$. Somatic pairing of chromosome 1 centromeres in interphase nuclei of human cerebellum. Hum Genet 1989;83 $231-4$.

7 Hopman AH, van Hooren E, van de Kaa C, Vooijs PG, Ramaekers FC. Detection of numerical chromosome aberrations using in situ hybridisation in paraffin sections of routinely processed bladder cancers. Mod Pathol 1991;4: 503-13.

8 Kim SY, Lee JS, Ro JY, Gay ML, Hong WK, Hittelman WN. Interphase cytogenetics in paraffin sections of lung tumors by non-isotopic in situ hybridisation. Mapping genotype/phenotype heterogeneity. Am f Pathol 1993;142: 307-17.

9 Tiainen M, Hopman A, Moesker O, Ramaekers F, Wessman $M$, Laasonen A, et al. Interphase cytogenetics on paraffin sections of malignant pleural mesothelioma. A comparison to conventional karyotyping and flow cytometric studies Cancer Genet Cytogenet 1992;62:171-9.

10 van de Kaa C, Nelson KA, Ramaekers FC, Vooijs PG, Hopman AH. Interphase cytogenetics in paraffin sections of routinely processed hydatidiform moles and hydropic abortions. $₹$ Pathol 1991;165:281-7.

11 van Dekken H, Kerstens HM, Tersteeg TA, Verhofstad AA, Vooijs GP. Histological preservation after in situ hybridisation to archival solid tumour sections allows discrimination of cells bearing numerical chromosome changes. $\mathcal{f}$ Patho 1992;168:317-24.

12 Segers K, Ramael M, Singh SK, van Daele A, Weyler J, van Marck E. Detection of numerical chromosome aberrations in paraffin-embedded malignant pleural mesothelioma by non-isotopic in situ hybridisation. $\mathcal{f}$ Pathol 1995;175: non-isotopi.

13 Harrison M, Magee HM, O'Loughlin J, Gorey TF, Dervan PA. Chromosome 1 aneusomy, identified by interphase cytogenetics in marnmographically detected ductal carcinoma in situ of the breast. $\mathcal{F}$ Pathol 1995;175:303-9.

14 Altman DG. Practical statistics for medical research. London Altman DG. Practical statistics

15 Wolman SR, Macoska JA, Micale MA, Sakr WA. An approach to definition of genetic alterations in prostate cancer. Diagnostic Molecular Pathology 1992;1:192-9.

16 Micale MA, Visscher W, Gulino SE, Wolman SR. Chromosomal aneuploidy in proliferative breast disease. Hum Pathol 1994;25:29-35. interpretation of large subpopulations, particu larly in which there has been chromosome gain. The assessment of chromosome loss is more difficult as "left shift" of the distribution 